\title{
COVID-19: risk for cytokine targeting in chronic inflammatory diseases?
}

Georg Schett ${ }^{1,2 凶}$, Michael Sticherling ${ }^{2,3}$ and Markus F. Neurath ${ }^{2,4}$

COVID-19, caused by the SARS-CoV-2 virus, has become pandemic. With sharply rising infection rates, patient groups characterized by an enhanced infection risk will be challenged by the virus. In this context, patients with chronic immune-mediated inflammatory diseases are of particular interest, as these diseases are characterized by an intrinsic immune dysfunction leading to inflammation that may enhance risk for severe infection.

Immune-mediated inflammatory diseases (IMIDs) often affect the inner and outer barriers of the body, such as the joints (rheumatoid arthritis (RA) and spondyloarthritis $(\mathrm{SpA})$ ), the gut (Crohn's disease (CD) and ulcerative colitis (UC)) and the skin (psoriasis ( $\mathrm{PsO}$ ) and atopic dermatitis (AD)). Treatment of IMIDs has developed rapidly in the past few years owing to better molecular profiling and the use of targeted interventions that neutralize disease-specific pro-inflammatory cytokines. In RA, the inhibition of TNF and IL-6R is effective, whereas SpA responds to inhibition of TNF and IL-17A. In CD and UC, TNF and IL-23 are the main effector cytokines. PsO is responsive to inhibition of TNF, IL-17A and IL-23, while inhibition of IL- 4 and IL-13 works in $\mathrm{AD}^{1}$ (FIG. 1). Despite differences in the target organs and the cytokine repertoires among individual IMIDs, commonalities exist, which are reflected by an exaggerated and sustained innate and adaptive immune response. In many forms of IMID, independent of the target organ or whether they are initiated via $T$ helper $1\left(\mathrm{~T}_{\mathrm{H}} 1\right), \mathrm{T}_{\mathrm{H}} 17$ or $\mathrm{T}_{\mathrm{H}} 2$ cell activation, infiltration of target tissues with large numbers of innate immune cells such as granulocytes and inflammatory macrophages is a hallmark.

Coronavirus disease 2019 (COVID-19), caused by the severe acute respiratory syndrome coronavirus 2 (SARS-CoV-2) virus ${ }^{2}$, leads to fast activation of innate immune cells, especially in patients developing severe disease. Circulating neutrophil numbers are consistently higher in survivors of COVID-19 than in non-survivors, and the infection also induces lymphocytopenia that mostly affects the $\mathrm{CD} 4^{+} \mathrm{T}$ cell subset, including effector, memory and regulatory $\mathrm{T}_{\text {cells }}{ }^{3}$. Reflecting innate immune activation, levels of many pro-inflammatory effector cytokines, such as TNF, IL- $1 \beta$, IL-6, IL-8, G-CSF and GM-CSF, as well as chemokines, such as MCP1, IP10 and MIP1 $\alpha$, are elevated in patients with COVID-19, with higher levels in those who are critically ill. In addition, the levels of some $T$ cell-derived cytokines, such as IL-17, are increased in the context of SARS-CoV-2 infection ${ }^{4}$.
SARS-CoV-2 infection drives a profound cytokine response in the host, comprising a series of mediators that are targeted in IMIDs (FIG. 1). In some patients with COVID-19, a cytokine storm develops that resembles secondary haemophagocytic lymphohistiocytosis, a hyperinflammatory state triggered by viral infections ${ }^{5}$.

Although the bulk of cytokines induced by SARS-CoV-2 infection as well as those being targeted in the various aforementioned IMIDs are important to mount inflammation, they do not seem to be essential for controlling virus clearance. Targeting IL-23 and IL-4/ IL-13 does not increase the risk for viral, bacterial or fungal infections, while inhibition of IL-17A only shows a signal for Candida species but not for viral infection. Targeting TNF and IL-6 increases the risk of bacterial infections but has lesser effects on viral infections (except for hepatitis B activation). Notably, although the incidence of influenza and the risk of developing complications from influenza infection are higher for patients with RA and CD, no signal associated with cytokine inhibitors was found ${ }^{6}$. Also, patients with RA or $\mathrm{CD}$ achieve normal immune responses to influenza vaccination when treated with anti-TNF agents, further supporting the concept that the effector cytokines induced by SARS-CoV-2 and targeted for treatment of IMIDs are critical for the inflammatory response but not for viral clearance ${ }^{7}$. Viral clearance seems to primarily depend on other cytokines such as IL-15, type I interferons and IFN $\gamma$.

Targeting pro-inflammatory cytokines with antibodies such as adalimumab, dupilumab, infliximab, ustekinumab, secukinumab and tocilizumab is clinical routine in IMIDs. Potential risk and benefits of cytokine inhibition need to be carefully addressed in order to recommend whether to continue or stop such treatments. Although at first sight cytokine inhibition might be considered as 'immune suppression' and therefore harmful in the context of the COVID-19 pandemic, these compounds neutralize individual mediators of the inflammation cascade rather than leading to broad immune 

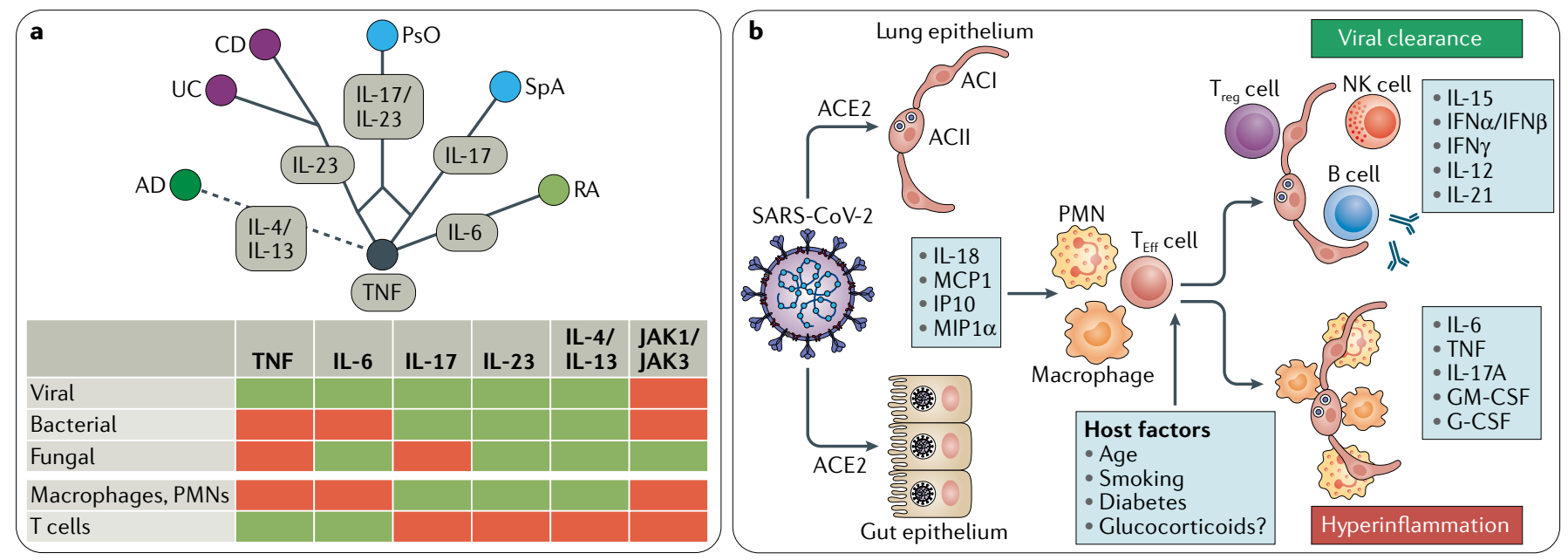

Fig. 1 | Cytokines in IMIDs and in COVID-19. a | A 'cytokine tree' of immune-mediated inflammatory diseases (IMIDs) showing their individual responsiveness to cytokine inhibitor therapy. The risk for viral, bacterial and fungal infections and effects on blood immune cells of the respective cytokine inhibition strategies are indicated below (red equals risk and green equals no risk). b| Cytokine pathogenesis of coronavirus disease 2019 (COVID-19). AC, alveolar cell; ACE2, angiotensinconverting enzyme 2; AD, atopic dermatitis; CD, Crohn's disease; JAK, Janus kinase; NK, natural killer; PMN, polymorphonuclear granulocyte; $\mathrm{PsO}$, psoriasis; $\mathrm{RA}$, rheumatoid arthritis; SARS-CoV-2, severe acute respiratory syndrome coronavirus 2; $\mathrm{SpA}$, spondyloarthritis; $\mathrm{T}_{\mathrm{EFF}}$ cell, $\mathrm{T}$ effector cell; $\mathrm{T}_{\text {req }}$ cell, regulatory T cell; $\mathrm{UC}$, ulcerative colitis.

suppression. On the other hand, cytokine inhibitors may mitigate the hyperinflammatory state, which is part of the pathogenesis of severe COVID-19. Indeed, studies using IL-6R and IL-6 inhibitors in COVID-19 have just been launched. Hence, approaches that do not affect viral clearance but inhibit hyperinflammatory host responses may exert beneficial effects in COVID-19.

Although targeting individual cytokines (TNF, IL-6, IL-17A, IL-23 or IL-4/IL-13), in contrast to glucocorticoids ${ }^{8}$, does not appear to increase viral infection rates or induce a more severe course of viral infection, the inhibition of multiple cytokines, for example, targeting interferon responses, may be different. Janus kinase (JAK) inhibitors, which target JAK1 and JAK3, developed for treatment of RA, CD and PsO show an increased risk for herpes zoster reactivation. Targeting JAK1 and JAK3 affects the function of several cytokines that are involved in antiviral responses such as type I interferons, IL-2, IL-15, IL-21 and IFN $\gamma$. Hence JAK1/JAK3 inhibitors could theoretically inhibit the clearance of SARS-CoV-2. On the other hand, JAK2 inhibition appears to block viral entry of SARS-CoV-2 and IL-17-induced cytokine activation ${ }^{9}$. Notably, IL-6 and GM-CSF, which are both induced by SARS-CoV-2, partly or fully depend on JAK2 signalling, suggesting that JAK2 could be a target in treating hyperinflammatory response in COVID-19.

At present, there is very limited experience on how COVID-19 affects patients with IMIDs treated with cytokine inhibitors. Nonetheless, a critical analysis of the role of pro-inflammatory cytokines in the pathophysiology of COVID-19 and of the risk of viral infection during anti-cytokine therapy suggests that most cytokine inhibitors may not automatically put patients with IMIDs at higher risk of developing severe COVID19. In accordance, most ad hoc recommendations from specialists in the fields of gastroenterology, rheumatology and dermatology do not support pre-emptively stopping anti-cytokine therapy if no signs of COVID19 are present. Remarkably, some cytokine inhibition strategies are currently being tested for the treatment of COVID-19, and hydroxychloroquine, a long-known drug used for the treatment of IMIDs, seems to show efficacy in COVID-19 (REF. ${ }^{10}$ ). Apart from these trials, IMID registers are currently being built up that will help to better understand the impact of COVID-19 in patients with autoimmune disease and to potentially uncover a protective role of certain cytokine inhibition strategies.

1. Schett, G. et al. How cytokine networks fuel inflammation: toward a cytokine-based disease taxonomy. Nat. Med. 19, 822-824 (2013).

2. Wu, F. et al. A new coronavirus associated with human respiratory disease in China. Nature 579, 265-269 (2020).

3. Wang, D. et al. Clinical characteristics of 138 hospitalized patients with 2019 novel coronavirus-infected pneumonia in Wuhan, China. JAMA 323, 1061-1069 (2020).

4. Huang, C. et al. Clinical features of patients infected with 2019 novel coronavirus in Wuhan, China. Lancet 395, 497-506 (2020).

5. Pedersen, S. F. \& Ho, Y. C. A storm is raging. J. Clin. Invest. https://doi.org/10.1172/JCI137647 (2020).

6. Blumentals, W. A., Arreglado, A., Napalkov, P. \& Toovey, S. Rheumatoid arthritis and the incidence of influenza and influenzarelated complications: a retrospective cohort study. $B M C$ Musculosskelet. Disord. 13, 158 (2012).

7. Andrisani, $G$. et al. Immune response to influenza $A / H 1 N 1$ vaccine in inflammatory bowel disease patients treated with anti TNF- $\alpha$ agents: effects of combined therapy with immunosuppressants. J. Crohns Colitis 7, 301-307 (2013).

8. Russell, C. D. Millar, J. E. \& Baillie, J. K. Clinical evidence does not support corticosteroid treatment for 2019-nCoV lung injury. Lancet 395, P473-P475 (2020).

9. Wu, D. \& Yang, X. O. TH17 responses in cytokine storm of COVID-19: an emerging target of JAK2 inhibitor fedratinib. J. Microbiol. Immunol. Infect. https://doi.org/10.1016/j.jmii.2020.03.005 (2020).

10. Chen, Z. et al. Efficacy of hydroxychloroquine in patients with COVID-19: results of a randomized clinical trial. Preprint at medRxiv https://doi.org/10.1101/2020.03.22.20040758 (2020).

Acknowledgements

The work is supported by the German Research Council (DFG: FOR2438/2886; SFB1181; TRR241), the German Ministry of Science and Education (project MASCARA), the European Union (ERC Synergy grant 4DnanoSCOPE) and EU/EFPIA Innovative Medicines Initiative 2 (project RTCure).

\section{Competing interests}

The author declares no competing interests. 